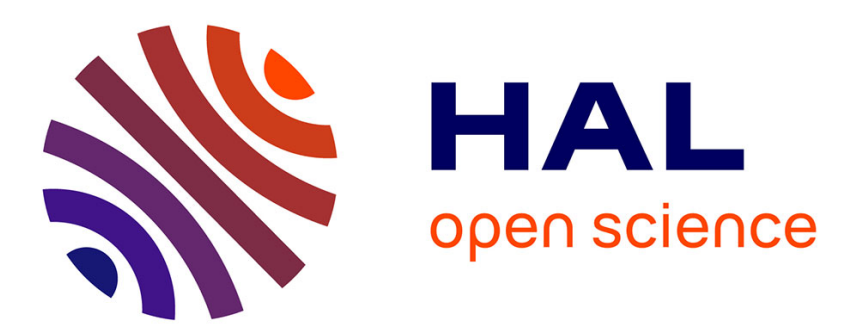

\title{
Analysis of frequency-shifting loops in integer and fractional Talbot conditions: electro-optic versus acousto-optic modulation
}

Hongzhi Yang, Marc Brunel, Marc Vallet, Haiyang Zhang, Changming Zhao

\section{- To cite this version:}

Hongzhi Yang, Marc Brunel, Marc Vallet, Haiyang Zhang, Changming Zhao. Analysis of frequencyshifting loops in integer and fractional Talbot conditions: electro-optic versus acousto-optic modulation. Journal of the Optical Society of America B, 2020, 37 (11), pp.3162-3169. 10.1364/josab.389801 . hal-03046194

\author{
HAL Id: hal-03046194 \\ https://hal.science/hal-03046194
}

Submitted on 15 Dec 2020

HAL is a multi-disciplinary open access archive for the deposit and dissemination of scientific research documents, whether they are published or not. The documents may come from teaching and research institutions in France or abroad, or from public or private research centers.
L'archive ouverte pluridisciplinaire HAL, est destinée au dépôt et à la diffusion de documents scientifiques de niveau recherche, publiés ou non, émanant des établissements d'enseignement et de recherche français ou étrangers, des laboratoires publics ou privés. 


\title{
Analysis of frequency-shifting loops in integer and fractional Talbot conditions: electro-optic vs acousto-optic modulation
}

\author{
Hongzhi YAng, ${ }^{1,2}$ MARc Brunel, ${ }^{*}$ MARc VAllet, ${ }^{3}$ Haiyang Zhang, ${ }^{1}$ \\ AND ChANGMing ZHAO ${ }^{1}$ \\ ${ }^{I}$ School of Optics and Photonics, Beijing Institute of Technology, Beijing, China \\ ${ }^{2}$ Qian Xuesen Laboratory of Space Technology, NO. 104 Youyi Road, Haidian district, Beijing, China \\ ${ }^{3}$ Univ Rennes, CNRS, Institut FOTON - UMR 6082, 35000 Rennes, France \\ *marc.brunel@univ-rennes1.fr
}

\begin{abstract}
Frequency-shifting loops (FSL) are analyzed theoretically in cases where the intracavity modulator induces two side-bands at each round-trip, a situation that can be commonly obtained with electro-optic intensity or phase modulators. Using a simple model, we discuss the ability of such loops to perform frequency-to-time mapping, in the integer Talbot condition, or pulse repetition rate enhancement, in the fractional Talbot condition. The results are compared to the established acousto-optic FSL with pure frequency shift. We show that, in spite of a more complicated situation resulting from the dual sideband modulation, pulse repetition rate amplification can be obtained with an amplitude modulator, and frequency-to-time mapping can be obtained with a phase modulator. This opens new routes to high-frequency manipulation of microwave-optical signals with high-bandwidth (multi-GHz) modulators.
\end{abstract}

(C) 2020 Optical Society of America under the terms of the OSA Open Access Publishing Agreement

\section{Introduction}

Frequency-shifted feedback loops (FSL) have attracted much attention recently because their peculiar time-frequency properties could lead to numerous applications, such as ultra-high repetition rate pulse generation [1], frequency-to-time mapping [2], optical signal processing [3], arbitrary waveform generation [4,5], heterodyne spectroscopy [6], and ranging [7,8,9]. Inspired from frequency-shifted feedback (FSF) lasers [10,11], a frequency-shifting loop is a sub-threshold ring resonator. It is typically seeded by a continuous-wave (cw) laser and it contains (i) a modulator that induces a frequency shift every round-trip, (ii) an optical amplifier to compensate for the losses, and (iii) an optical filter. Up to now the modulator in the FSL was usually an acousto-optic (AO) frequency-shifter. While it features high frequency conversion efficiency in the sub-100 MHz range, AO has limited efficiency in the $\mathrm{GHz}$ range and offers limited tunability. Besides the AO frequency-shifter, a phase modulator (PM) or an amplitude modulator (AM) can also provide frequency-shifted sidebands at each round-trip, with a potentially much higher bandwidth. In this respect, electro-optic (EO) modulators have been demonstrated to provide high repetition rates [12] or peculiar doublepulse [13] regimes. In addition to high modulation frequency and bandwidth, EO modulators are compact and easy to integrate with other fiber devices. In view of enhancing the properties of FSLs in terms of bandwidth and tunability for applications, it is hence important to analyze the properties of EO-based FSLs.

One striking feature of AO-FSL is its ability to convert a $\mathrm{cw}$ narrow linewidth seed into Fourier-transform-limited pulses. This property is linked to the temporal Talbot effect [14, 15]. It permits to generate pulses at a repetition rate equal to the round-trip rate, when the frequency-shift equals this round-trip rate (the integer Talbot condition), or to a multiple of the round-trip rate (the fractional Talbot condition). In addition, when the integer Talbot 
condition is met, the output temporal trace maps the input optical spectrum, leading to optical real-time Fourier processing of RF signals [2]. While theoretical models have been proposed to explain the frequency-to-time mapping (FTM) and temporal fractional Talbot effect in the AO-based configuration, the electro-optic modulator needs further investigation, because it generates two sidebands at each round-trip. Double-pulse operation was reported in [13], but frequency-to-time mapping or fractional Talbot condition was not considered. Moreover phase modulation has never been studied in this context, to the best of our knowledge. It is hence the aim of this paper to propose a general time-delay interference model suited to any type of modulator, and to compare the properties of EOM-based modulators with either amplitude or phase modulation, to the AO-FSL. In this study we put the emphasis on investigating the properties in the integer and fractional Talbot conditions.

In Section 2 we recall the FSL principles and derive the general analytical framework, introducing a loop modulation function. Then, we recall the basics of AO-FSLs for completeness (Section 3), and then focus on amplitude-modulated (Section 4) and phasemodulated EOM-FSL (Section 5). The potentials of EO-FSLs are discussed in the conclusion (Section 6).

\section{General time-delayed interference model}

The frequency-shifting loop is modeled theoretically by using a time-delayed interference model that takes the modulation function of the modulator, round-trip delay and optical amplifier into account. The general setup of the FSL is shown in Fig. 1. We assume that the loop is seeded by an external cw laser, whose electric field is $E_{i n 1}=E_{0} e^{-j \omega_{0} t}\left(\omega_{0}\right.$ is the optical frequency) at the coupler input. The output field $E_{\text {out }}$ writes:

$$
\left[\begin{array}{l}
E_{\text {out } 1} \\
E_{\text {out } 2}
\end{array}\right]=\left[\begin{array}{ll}
t_{11} & t_{12} \\
t_{21} & t_{22}
\end{array}\right]\left[\begin{array}{l}
E_{\text {in } 1} \\
E_{\text {in } 2}
\end{array}\right]
$$

where $\left[t_{i j}\right]$ is the transmission matrix of the coupler. Introducing a constant real parameter $\gamma$ that includes loss and gain factors inside the loop, the modulation function of the modulator $\gamma(t)$, and the round-trip time $\tau$, the electric field at output port 2 writes:

with

$$
E_{\text {out } 2}(t)=t_{21} E_{\text {in } 1}+t_{22} E_{\text {in } 2}=t_{21} E_{0} e^{-j \omega_{0} t}+t_{22} \gamma E_{\text {out } 2}(t-\tau) Y(t-\tau),(2)
$$

$$
E_{\text {out } 2}(t-\tau)=t_{21} E_{0} e^{-j \omega_{0}(t-\tau)}+\mathrm{t}_{22} \gamma E_{\text {out } 2}(t-2 \tau) \gamma(t-2 \tau),
$$

and so on. Combination of $\mathrm{N}$ round-trips yields:

$$
E_{\text {out } 2}(t)=t_{21} E_{0} e^{-j \omega_{0} t}+t_{21} \sum_{l=1}^{N} t_{22}^{l} \gamma^{l} \prod_{n=1}^{l} \gamma(t-n \tau) E_{0} e^{-j \omega_{0}(t-l \tau)} .
$$



Fig.1 Sketch of the FSL. The modulator induces pure frequency-translation (AO), or dualsideband generation in either amplitude (EO-AM), or phase (EO-PM) cases.

The experimentally accessible and useful signal is at the output port 1 , where

$$
E_{\text {out } 1}(t)=\left[t_{11}+\frac{t_{21} t_{12}}{t_{22}} \sum_{l=1}^{N} \bar{\gamma}^{l} \prod_{n=1}^{l} \Upsilon(t-n \tau)\right] E_{0} e^{-j \omega_{0} t}
$$


where $\bar{\gamma}=t_{22} \gamma e^{j \omega_{0} \tau}$ is a constant complex parameter containing the loop optical phase as well as the gain and loss parameters. In the following we focus our discussion on the loop function $L(t)$ defined by

$$
L(t)=\sum_{l=1}^{N} \bar{\gamma}^{l} \prod_{n=1}^{l} \Upsilon(t-n \tau) .
$$

$L(t)$ is essentially the part of the output complex optical envelope generated in the loop, including all the generated sidebands. We will investigate three different cases that can lead to a frequency-shift $f_{m}=\omega_{m} / 2 \pi$. Modulation functions of the three different modulators can be written as follows:

$$
\begin{gathered}
\Upsilon_{F}(t)=e^{j \omega_{m} t}, \\
\Upsilon_{A}(t)=\sin \left[\Gamma_{0}+\Gamma_{m} \sin \left(\omega_{m} t\right)\right], \\
\Upsilon_{\phi}(t)=e^{j \delta \sin \left(\omega_{m} t\right)},
\end{gathered}
$$

corresponding to the frequency translation (AO), the amplitude modulator (EO-AM), and the phase modulator (EO-PM), respectively. In Eq. $(7 \mathrm{~b}), \Gamma_{0}$ is a static retardance, and $\Gamma_{\mathrm{m}}$ is the modulation depth. In Eq. (7c), $\delta$ is the modulation depth of the phase modulator. The purpose of our analysis is to derive the different characteristics of the loop functions $L_{F}, L_{A}, L_{\phi}$ in integer and Fractional Talbot conditions, and hence to predict the ability of either FSL scheme to perform harmonic repetition rate pulse trains or frequency-to-time mapping. In all cases the optical power at port 1 , that is $P_{\text {out }}(t)=\left|E_{\text {out } 1}(t)\right|^{2}$, will be obtained by

$$
P_{\text {out }}(t)=\left|t_{11}+\frac{t_{21} t_{12}}{t_{22}} L(t)\right|^{2} P_{0} .
$$

While the AO-FSL case has already been investigated in detail by Guillet de Chatellus et al. [1-3], the EO-FSLs need to be analyzed. Since our formulation is slightly different from the one adopted before, we begin the next sections by considering quickly this most-well-known case in order to point out the differences with the EO-FSL following results.

\section{Frequency translation (AO-FSL)}

Using Eq. 7(a) in Eq. 6, the loop function is $L_{F}(t)=\sum_{l=1}^{N} \bar{\gamma}^{l} \prod_{n=1}^{l} e^{j \omega_{m}(t-n \tau)}$. In the integer Talbot condition that writes $f_{m} \tau=f_{m} / f_{c}=p$, with $p \in \mathbb{N}^{*}$, we have $e^{j \omega_{m}(t-n \tau)}=e^{j \omega_{m} t}$, then $L_{F}(t)=\sum_{l=1}^{N} \bar{\gamma}^{l} e^{j l \omega_{m} t}$. In the limit $N \rightarrow \infty$, this sum of a geometric series writes:

$$
L_{F}(t)=\frac{t_{22} \gamma e^{j \omega_{m} t} e^{j \omega_{o} \tau}}{1-t_{22} \gamma e^{j \omega_{m} t} e^{j \omega_{O} \tau}},
$$

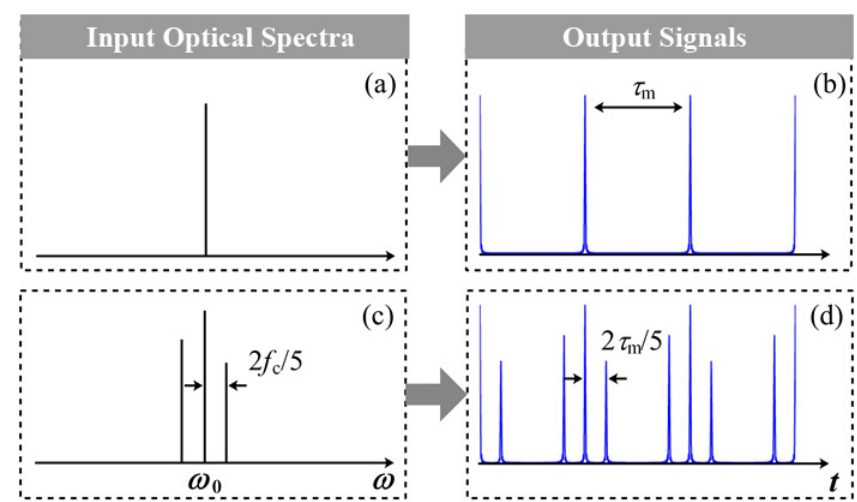

Fig.2 FTM process in the AO-FSL. (a) Single-frequency input yields the pulsed output in (b). (c) Multi-tone input yields the output signal in (d) that maps the input spectrum. 
where we have expanded $\bar{\gamma}$ in order to emphasize the role of the optical phase $\omega_{0} \tau$. Since $t_{22} \gamma<1$ (assuming $t_{22}$ real positive), obviously $L_{F}(t)$ will be a sharp function peaked in $\omega_{m} t+\omega_{0} \tau=2 k \pi(k \in \mathbb{Z})$. Therefore, the response of the loop to a single optical frequency (cw seed) is a periodic function whose repetition rate is $f_{m}$, as shown for example in Fig. 2(a)-(b). Moreover, it is important to notice here that the pulse positions in time are highly dependent on the optical frequency of the seed laser, through the term $e^{j \omega_{o} \tau}$. If the seed laser contains multiple frequencies $\omega_{i}$, as shown in Fig. 2(c)-(d), the output traces in the time domain will map the input spectrum, each optical frequency yielding maxima when $\omega_{m} t+\omega_{i} \tau=2 k \pi(k \in \mathbb{Z})$. Therefore, the response of the loop is a time map of the input spectrum, repeated at the fundamental loop frequency $f_{c}$. The resolution of the process was evaluated in [2] to be $\delta f=\frac{f_{m} f_{c}}{\Delta f} \sim \frac{f_{c}}{N}$ where the loop bandwidth is $\Delta f \sim N f_{m}$.

Now under the fractional Talbot condition, i.e., when $f_{m} \tau=f_{m} / f_{c}=p / q$, with $(p, q)$ coprimes, the modulation function becomes $\gamma_{F}(t-n \tau)=e^{j \omega_{m} t-j 2 \pi n \frac{p}{q}}$. Then $L_{F}(t)$ writes

$$
L_{F}(t)=\sum_{l=1}^{N} \bar{\gamma}^{l} \prod_{n=1}^{l} e^{j\left(\omega_{m} t-2 \pi n \frac{p}{q}\right)} .
$$

Eq. (10) yields different analytical expressions depending on the parities of $p$ and $q$. For the analytical derivation we choose $N=K q\left(K \in \mathbb{N}^{*}\right)$. Defining $f^{(l)}(t)=\bar{\gamma}^{l} \prod_{n=1}^{l} e^{j \omega_{m} t-j 2 \pi n \frac{p}{q}}$ and $\rho=\left[\bar{\gamma} e^{j \omega_{m} t}\right]^{q} e^{j \pi(q+1)}$, one can then show (see Appendix A) that

$$
L_{F}(t)=\frac{1-\rho^{K}}{1-\rho} \sum_{l=1}^{q} f^{(l)}(t),
$$

which we now expand according to the parity of $q$.

(i) Let $q$ odd. By expanding $\rho$, the loop function writes:

$$
L_{F}(t)=\frac{1-\left(t_{22} \gamma\right)^{N} e^{j N \omega_{o} \tau} e^{j N \omega_{m} t}}{1-\left(t_{22} \gamma\right)^{q} e^{j q \omega_{o} \tau} e^{j q \omega_{m} t}} \sum_{l=1}^{q} f^{(l)}(t) .
$$

Hence the output trace tends to a modulated function with intensity peaks localized at values defined by $q \omega_{o} \tau+q \omega_{m} t=2 k \pi(k \in \mathbb{Z})$. This behavior corresponds to the generation of $q$ optical pulses per period. In the time domain, this corresponds to a repetition rate equal to $q f_{m}=p f_{c}$.

(ii) Let $q$ even. Expanding $\rho$, the loop function becomes:

$$
L_{F}(t)=\frac{1-(-1)^{K}\left(t_{22} \gamma\right)^{N} e^{j N \omega_{o} \tau} e^{j N \omega_{m} t}}{1+\left(t_{22} \gamma\right)^{q} e^{j q \omega_{0} \tau} e^{j q \omega_{m} t}} \sum_{l=1}^{q} f^{(l)}(t)
$$

As for Eq. (12), the output results in a peaked function localized at values of $q \omega_{o} \tau+q \omega_{m} t=(2 k+1) \pi,(k \in \mathbb{Z})$. There are $q$ pulses per modulation period and the repetition rate is $q f_{m}=p f_{c}$ but, at variance with case (i), the pulses are shifted from the origin by a delay $\pi / q$.

In order to illustrate the fractional Talbot effect in this AO-FSL, we evaluate Eq. (8) with modulation frequencies corresponding to the cases of Eqs. (12)-(13). We choose simulation parameters as follows: the fundamental loop frequency is $f_{c}=10 \mathrm{MHz}(\tau=100 \mathrm{~ns})$, the frequency shift is adjusted to $f_{m}=10 f_{c}+\left(p^{\prime} / q^{\prime}\right) f_{c}$. The splitting ratio of the $2 \times 2$ coupler is 50:50. The loop loss is 0.4 and the gain is 3.5. We fix the round-trip number $N=100$. In Fig. 3 , we report the pulse trains obtained when $p^{\prime} / q^{\prime}=5 / 4,12 / 11,16 / 15$, and $21 / 20$, that is $p / q$ $=45 / 4,122 / 11,166 / 15$, and 221/20. As shown by Fig. 3, these correspond to $q f_{m}$-repetition rates ranging here from $4 f_{m}$ to $20 f_{m}$. These simulations confirm the analytical predictions: the loop generates periodic pulses with a repetition rate $q f_{m}=p f_{c}$, with a time-shift depending on the parity of $q$. Obviously the condition $N=K q$ is not respected in all these cases, but we checked that this has actually little influence on the waveforms; in other words, the harmonics higher than $K q$ are negligible. 
After having revisited the AO-based FSL for which these results had already been observed, we now turn to dual-sideband EO cases, first in the amplitude-modulation (AM) scheme that was recently shown to lead to pulse doublets [13], and then in the phasemodulation (PM) regime.



Fig.3 Fractional Talbot effect for the AO-FSL with $p / q$ ratios as indicated. The resulting pulse repetition rate is $q f_{m}$ (with $f_{m}$ close to $110 \mathrm{MHz}$ in all these examples).

\section{EO-FSL with amplitude modulation}

By using Eq. (7b) in Eq. (6) we write the loop function $L_{A}(t)=\sum_{l=1}^{N} \bar{\gamma}^{l} \prod_{n=1}^{l} \sin \left(\Gamma_{0}+\right.$ $\left.\Gamma_{m} \sin \left(\omega_{m}(t-n \tau)\right)\right)$.

\subsection{Integer Talbot condition}

When $f_{m} \tau$ is an integer, we have $\sin \left(\omega_{m}(t-n \tau)\right)=\sin \left(\omega_{m} t\right)$. Then the loop function becomes

$$
L_{A}(t)=\sum_{l=1}^{N} \bar{\gamma}^{l}[\sin \theta(t)]^{l}=\frac{t_{22} \gamma e^{j \omega_{o} \tau} \sin \theta(t)}{1-t_{22} \gamma e^{j \omega_{o} \tau} \sin \theta(t)},
$$

where $\theta(t)=\Gamma_{0}+\Gamma_{m} \sin \left(\omega_{m} t\right)$. Note that the $e^{j \omega_{m} t}$ term of the AO case in Eq. (9) is replaced by $\sin \theta(t)$, resulting in a very different behavior. Considering the special case where $e^{j \omega_{o} \tau}=1$, Eq. (14) shows here that the loop delivers a pulse every time $\theta(t)=$ $\pi / 2+2 k \pi(k \in \mathbb{Z})$. Interestingly, two temporally separated solutions appear in one modulation period. According to this analysis, we may predict that the output of the amplitude-modulated loop in the time domain delivers a periodic series of pulse doublets [13]. Moreover, the output signal does not map the input spectrum anymore. This is evidenced by plotting the output signals obtained with different input spectra, as depicted in Fig. 4. The output time traces in the amplitude-modulated FSL show the same patterns (double pulses) regardless of the input optical spectra. Note that $e^{j \omega_{o} \tau}=-1$ leads to $\theta(t)=-\pi / 2+2 k \pi$ and to the same conclusions. Besides, if the condition $e^{j \omega_{o} \tau}= \pm 1$ is not met, we verified numerically that the output modulation amplitude is much weaker, and there is no FTM a fortiori. This emphasizes an important difference from the AO-FSL: it means that an EO-AM FSL cannot be used for real-time Fourier transformation.

\subsection{Fractional Talbot condition}

We now consider the fractional Talbot condition $f_{m} \tau=f_{m} / f_{c}=p / q$, with $(p, q)$ co-primes, in order to check for the possibility of using this scheme for harmonic high-repetition rate pulses. The equations are more cumbersome than in the AO-FSL case. However, there are some conditions under which the loop function can be simplified. To this aim, we choose the static phase retardance $\Gamma_{0}=0$ (a case corresponding to carrier suppression), and we assume 
that the modulation is weak enough to write $\sin \theta(t) \approx \theta(t)$. Then $\gamma(t-n \tau)=$ $\Gamma_{m} \sin \left(\omega_{m} t-2 \pi n p / q\right)$ is used to write the loop function as

$$
L_{A}(t)=\sum_{l=1}^{N} \bar{\gamma}^{l}\left(\Gamma_{m}\right)^{l} \prod_{n=1}^{l} \sin \left(\omega_{m} t-2 \pi n \frac{p}{q}\right) .
$$

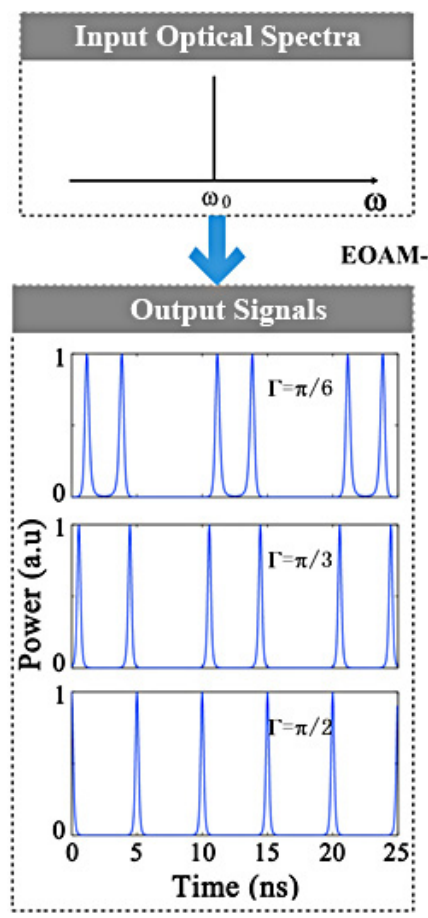

\section{Input Optical Spectra}



\section{Output Signals}

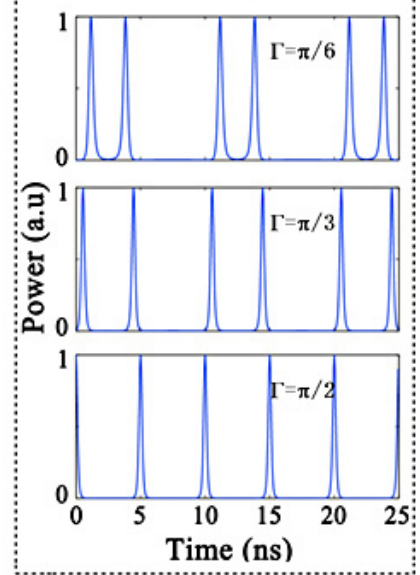

Fig. 4. EO-AM FSL under the integer Talbot condition. Simulation output power with (left) single frequency and (right) multi-tone input. Double-pulse operation depends on the EO bias $(\Gamma)$ but there is no frequency-to time mapping.

We could not find any simpler expression of Eq. (15) that could be directly compared with Eq. (11). However, simulations were performed using Eq. (8) with different fractional orders $p / q$ in Eq. (15). All simulation parameters are the same as before, except for the loop gain that is now set to 5.3. As illustrated in Fig. 5, the output traces show trains of pulses with repetition rates $q f_{m}=p f_{c}$ (fractional Talbot effect) whatever the parity of $q$. Meanwhile, the pulse train amplitude is modulated at $f_{m}$.
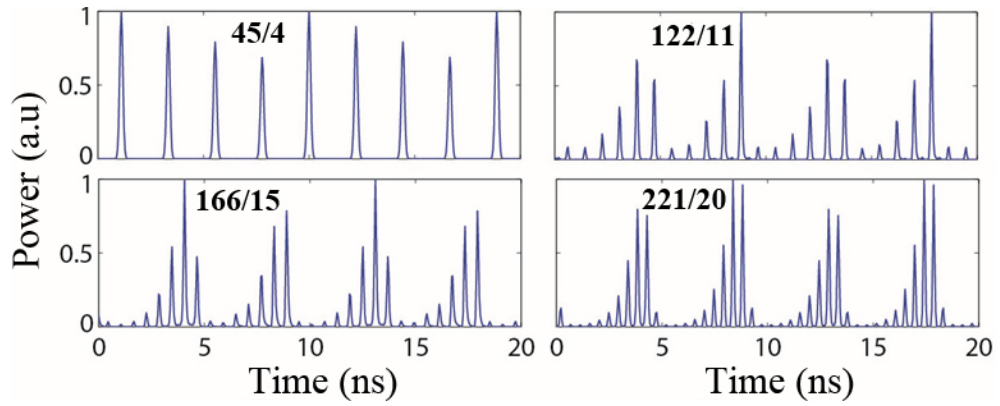

Fig. 5. EO-AM FSL under fractional Talbot condition with $p / q$ ratios as indicated. The resulting pulse repetition rate is $q f_{m}$ (with $f_{m}$ close to $110 \mathrm{MHz}$ in these examples). Note the $1 / f_{m}$-periodic modulation amplitude. 
In conclusion of this section, the EO-based FSL with amplitude modulation presents also the fractional Talbot property already found in the pure frequency shifting AO case, but with additional amplitude modulation. Besides, it is also found that the integer Talbot condition yields fundamental pulse trains, but the loop function do not depend on the optical frequency any more, which means that the input spectrum is not mapped in the time domain. In order to complete our study, we now study the last case concerning EO-based phase modulation.

\section{EO-FSL with phase modulation}

Inserting Eq. (7c) in Eq. (6) yields the loop function $L_{\phi}(t)=\sum_{l=1}^{N} \bar{\gamma}^{l} \prod_{n=1}^{l} e^{j \delta \sin \left[\omega_{m}(t-n \tau)\right]}$.

\subsection{Integer Talbot condition}

Under the integer Talbot condition $f_{m} \tau=p\left(p \in \mathbb{N}^{*}\right)$, we find $e^{j \delta \sin \left[\omega_{m}(t-n \tau)\right]}=e^{j \delta \sin \left(\omega_{m} t\right)}$. Then the geometric series of $L_{\phi}(t)$ can be easily calculated:

$$
L_{\phi}(t)=\frac{t_{22} \gamma e^{j \theta(t)}}{1-t_{22} \gamma e^{i \theta(t)}}
$$

where we introduced

$$
\theta(t)=\delta \sin \left(\omega_{m} t\right)+\omega_{0} \tau .
$$

Similarly to the situation found with the AO in Section 2 (see Eq. (9)), $L_{\phi}(t)$ is a function peaked in $\theta(t)=2 k \pi(k \in \mathbb{Z})$, with a repetition rate $f_{m}$. From Eq. (16) we see that the optical frequency will play a role, as in the AO case. However the $\left(\omega_{m} t\right)$ term of Eq. (9) is replaced here by $\delta \sin \left(\omega_{m} t\right)$. In order to understand the consequences of this change, we have to discuss the solutions of $\theta(t)=2 k \pi$ under various modulation conditions: $\delta=\pi, \delta>\pi$, and $\delta<\pi$.

i) Phase modulation depth $\delta=\pi$.

An illustration of $\theta(t)=2 k \pi$ is provided in Fig. 6 under different input spectral situations, leading to the following comments. First, one single optical frequency (blue curve in Fig. 6(a) for example) will induce a doublet of pulses per period, in the same fashion as the AM case of section 4. Second, a narrow bandwidth spectrum will result in a peculiar frequency-to-time mapping, where the input spectrum and its mirror image will be mapped in one period (red and blue curves in Fig. . 6(a). Third, it is worthwhile to note that, due to the $\left(\omega_{0} \tau\right)$ term in Eq. (17), two optical frequencies separated by multiples of the fundamental frequency $f_{m}=1 / \tau$ would produce identical time traces, as shown in Fig. 6(b). Hence, to ensure that the FTM process is achieved unambiguously, the frequency bandwidth of the seed

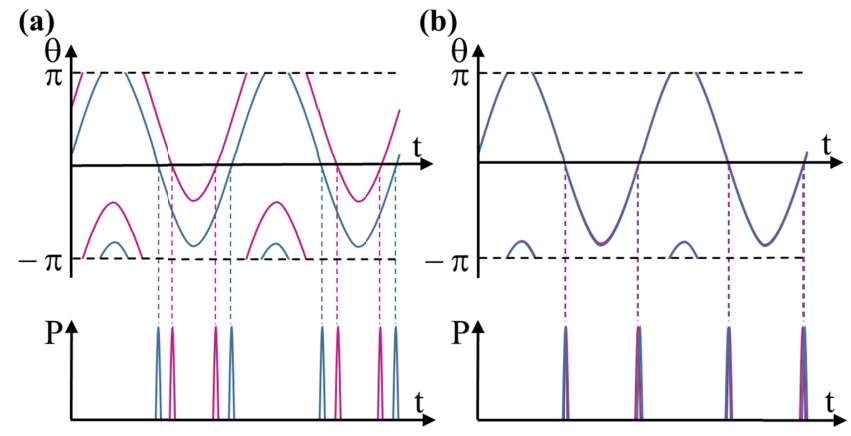

Fig. 6 EO-PM case. Sketch of the double-pulse operation with frequency-to-time mapping when $\delta=\pi$. (a) FTM with spectral mirror effect; (b) Two-tone input (frequency difference $f_{c}$ ) showing the ambiguity. 
laser should be kept smaller than $f_{c}$. Fourth, one can estimate the resolution $\Delta f$ of the process. In the linear part of the sine function we find a situation similar to the AOFS case discussed in [2]. The input frequency and output pulse time are linearly related, and two input lines spectrally separated by $\Delta f$ yield pulses separated by $\Delta t$ with $\delta \times \omega_{m} \Delta t=2 \pi \Delta f \tau$. Then, by considering that a single-frequency input yields an output pulse-width given by $\Delta t \sim 1 / 2 N f_{m}$ ( $2 N$ in-phase spectral lines in the loop), one finds the resolution $\Delta f \sim \delta \times \frac{f_{m} \Delta t}{\tau}=\frac{\delta}{2} \frac{f_{c}}{N}$. The resolution depends notably on the modulation depth but, as a rule of thumb, one may retain:

$$
\Delta f \sim \frac{f_{c}}{N} .
$$

It is interesting to note that the resolution scales as $1 / N$, and it can be improved by enhancing the number of spectral lines in the loop, as in the AOFS case.

Frequency to time mapping is simulated by using Eqs. (16)-(17) in Eq. (8), keeping the same parameters as in previous Section. For illustration, we use a three-tone input, like a carrier with two intensity un-balanced sidebands, as shown in Fig. 7(c). Due to the sinusoidal modulation function of the phase-modulating EOM, each optical frequency maps to a doublepulse in the time domain. Fig. 7(d) then exhibits the "mirror effect". Because of this property, the output traces with different optical frequencies are distributed in the half period, which is quite similar to a traditional FFT process. Also the loop fundamental frequency corresponds to the sampling frequency of the FFT process, limiting the highest frequency of the sampling signal. Besides, we find in the simulations that when the integer Talbot condition is met $\left(f_{m} / f_{c}=p\right)$ and $t_{22} \gamma$ is fixed, the ratio of the pulse width to the pulse period (duty cycle) is always the same regardless of the value of $p$, as predicted by Eq. (18). Finally, it is important to note that real-time Fourier transform can be obtained only provided that the solutions of Eq. (17) stay in the linear region of the sine function. Conversely, when the solutions are out of the linear region, this EO-PM configuration performs nonlinear FTM.

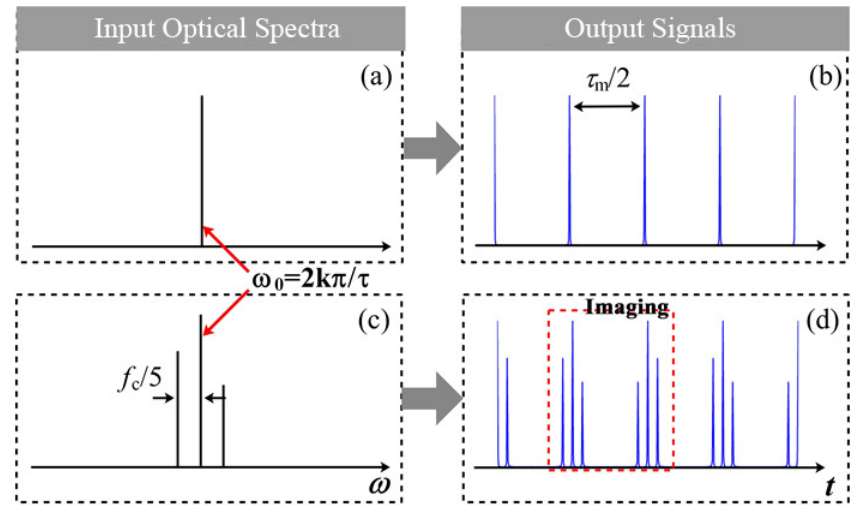

Fig. 7. FTM of the EO-PM FSL when $\delta=\pi$. (a),(c) input laser spectra; (b),(d) corresponding output signals.

\section{ii) Phase modulation depth $\delta>\pi$.}

When the modulating depth is larger than $\pi$, the response of the phase-modulated FSL to a single optical frequency is shown in Fig. 8. As the optical frequency of the seed laser changes, the solutions of $\theta(t)=2 k \pi$ may extend over different possible values of $k$, which is illustrated in Fig. 8(b). In conclusion the single-frequency seed laser can yield a 2-pulse or a 4-pulse regime in one modulating period.

In order to validate the analysis, we simulate the output traces when $\delta=5 \pi / 4$. As an example we take three different optical frequencies: $f_{0}\left(\omega_{0} \tau=2 k^{\prime} \pi, k^{\prime}\right.$ is an integer), $f_{0}+1 / 5 f_{c}, f_{0}+2 / 5 f_{c}$, and then feed Eq. (8) with Eq. (16). As illustrated in Fig. 9(a), 
solutions of $\delta \sin \left(\omega_{m} t\right)+\omega_{o} \tau=2 k \pi$ appear with adjacent values of $k$. One frequency $f_{0}+2 / 5 f_{c}$ is mapped to four pulses in one period, while the two other tones map to two pulses. The "mirror effect" appears as in the preceding $(\delta=\pi)$ case.

(a)

(b)

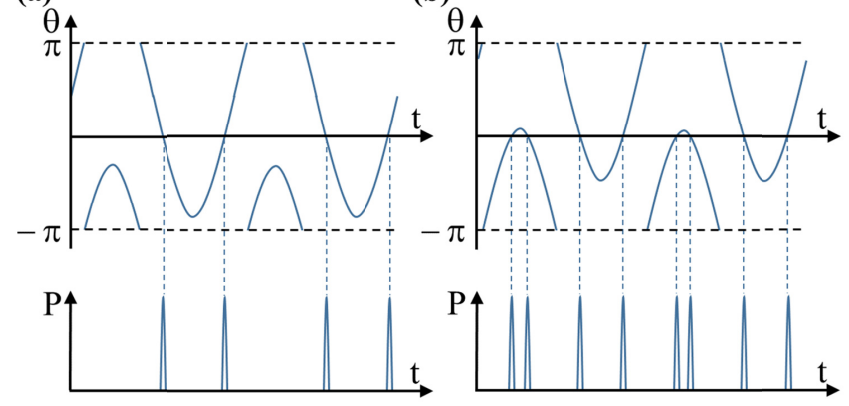

Fig. 8. EO-PM case Sketch of the response when $\delta>\pi$. Depending on the loop parameters $(\delta, \tau)$, the response of the FSL to a single frequency seed can be (a) double-pulse; or (b) fourpulse generation.
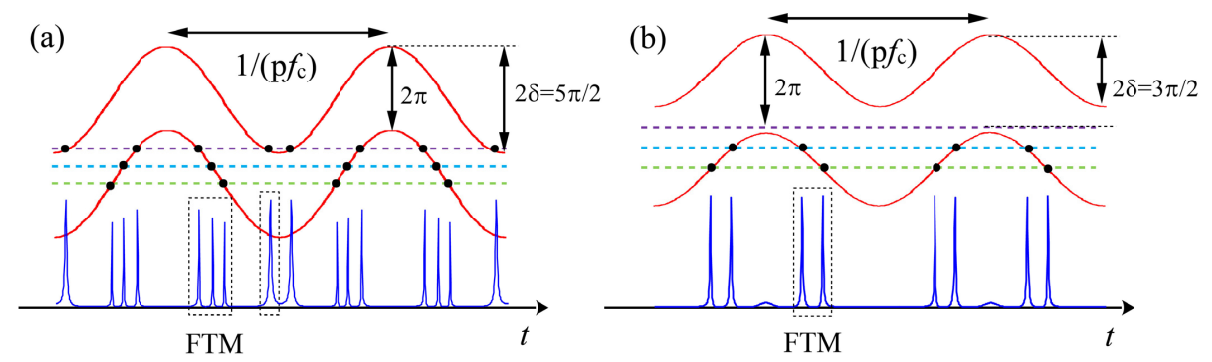

Fig. 9. Simulations of the FTM with a three-tone input. (a) $\delta=5 \pi / 4$; (b) $\delta=3 \pi / 4$.

(a)

(b)

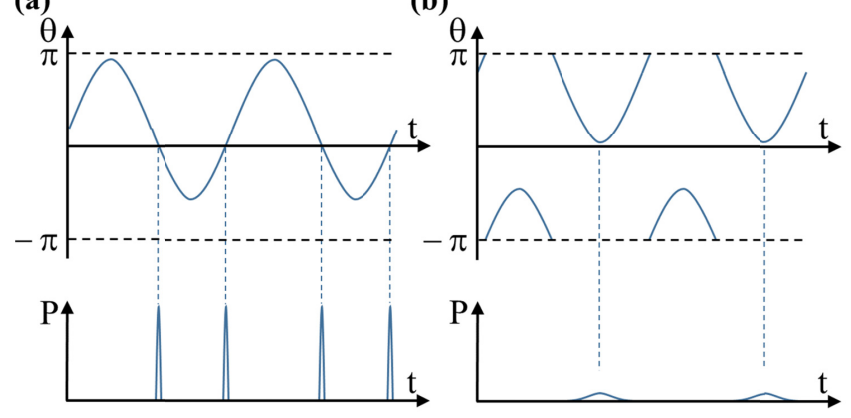

Fig. 10. Sketch of the FSL response when $\delta<\pi$ with different values of $\omega_{o} \tau$. (a) pulse generation or (b) reduced interference when the condition $\theta(t)=2 k \pi$ cannot be met.

iii) Phase modulation depth $\delta<\pi$.

Finally, when the modulating depth is less than $\pi$, the response of the phase-modulated FSL to the optical frequency of the seed laser is shown in Fig. 10. At variance with the preceding cases, possibly there is not any solution $\theta(t)=2 \mathrm{k} \pi$. Depending on the value of $\omega_{0} \tau$, one optical frequency induces either a pulse doublet regularly mapped along the timeaxis, as shown in Fig. 10(a), or a partial interference leading to a smaller intensity maximum as shown in Fig. 10(b). This is confirmed by the simulation that we perform with the same input as before, leading to the output trace of Fig. 9(b). 


\subsection{Fractional Talbot condition}

Under the fractional Talbot condition, when $f_{m} / f_{c}=p / q,(p, q)$ co-primes, Eq. (5) is given by $L_{\phi}(t)=\sum_{l=1}^{N} \bar{\gamma}^{l} \prod_{n=1}^{l} e^{j \delta \sin \left(\omega_{m} t-2 \pi n \frac{p}{q}\right)}$, and can be written:

$$
L_{\phi}(t)=\sum_{l=1}^{N} \bar{\gamma}^{l} e^{j \delta \sum_{n=1}^{l} \sin \left(\omega_{m} t-2 \pi n \frac{p}{q}\right)} .
$$

From the derivation given in Annex $\mathrm{B}$, assuming $N=K q\left(K \in \mathbb{N}^{*}\right)$, we find that

$$
L_{\phi}(t)=\frac{1-\bar{\gamma}^{N}}{1-\bar{\gamma}^{q}} \sum_{l=1}^{q} f^{(l)}(t),
$$

where $f^{(l)}(t)=\bar{\gamma}^{l} \prod_{n=1}^{l} e^{j \delta \sin \left(\omega_{m} t-2 \pi n p / q\right)}$. There is an important point to observe here: while it is formally similar to Eq. (11), Eq. (20) shows that the high-order harmonics are eliminated from the FSL under the fractional Talbot condition. The $\operatorname{sum} \sum_{l=1}^{q} f^{(l)}(t)$ contains the $q$ first round-trips in the loop only, so that higher-than- $q$ harmonics, if any, would come from the pre-factor in Eq. (20). Contrary to Eq. (11) where the pre-factor contains $e^{j N \omega_{m} t}$ components, here the pre-factor is a constant value, leaving no trace of high-order harmonics. This analysis is confirmed by simulations: We plot the output waveforms with different values of $p$ and $q$, still with the same parameters as before. Fig. 11 shows the simulated output waveforms with $p / q=45 / 4, p / q=122 / 11, p / q=166 / 15$ and $p / q=$ 221/20. Compared with the fractional Talbot laser based on the AO-FSL (Fig. 3) and the EOAM-FSL (Fig. 5), the output signals present smooth waveforms, without the highharmonic content leading to high-repetition rate pulses. We checked that the FFTs of the signals in Fig. (11) present appreciable harmonics up to $q^{\text {th }}$ order only.

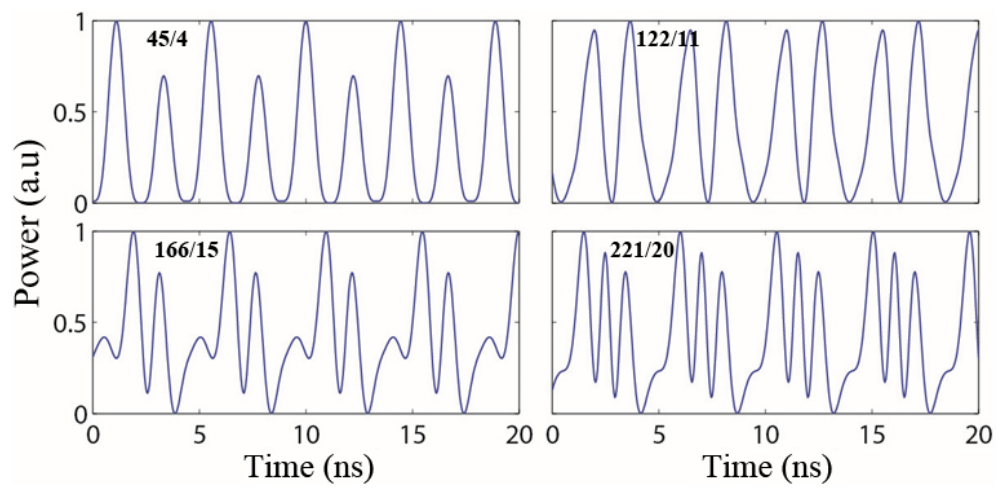

Fig. 11. EO-PM FSL in the fractional Talbot condition. Output waveforms with $p / q$ ratios as indicated.

\section{Conclusion}

We have derived analytical expressions to investigate the output characteristics of the phase, amplitude and frequency-modulating FSF loops. These expressions are deduced from a linear time-delayed interference model. We emphasized in this analysis the cases of the integer and fractional Talbot conditions, and compared the EO-AM and EO-PM schemes to the AO scheme. We can draw some conclusions about the ability of either EO-AM or EO-PM schemes to provide frequency-to-time mapping (FTM), or to multiply the repetition rate through the fractional Talbot effect

When the integer Talbot condition is satisfied $\left(f_{m} / f_{c}=p\right)$, the output signals show periodic pulses in any case, with a $f_{m}$ repetition rate. However, the three modulation schemes have specific properties. On the one hand, amplitude modulation (EO-AM) FSL fails to provide FTM but features a double-pulse regime with an interval between the two pulses that can be continuously adjusted by changing the static phase retardance or the modulation depth. 
On the other hand, phase modulation (EO-PM) can yield FTM, with an interesting "mirror effect" due to the sine modulation function in one modulation period. We find a possible FTM ambiguity, which could be mitigated by adjusting the modulation depth. Another specific feature of the EO-PM is the possibility of nonlinear FTM that may lead to chirped pulse generation.

When the fractional Talbot condition is met $\left(f_{m} / f_{c}=p / q\right)$, the frequency-translation (AO) and amplitude-modulation (EO-AM) FSLs share the ability to multiply the repetition rate of the periodic pulses, with $q f_{m}=p f_{c}$. The AM case has additional amplitude modulation of the high-repetition-rate pulse train. On the other side, a phase-modulated FSL cannot show the temporal fractional Talbot effect due to the destructive interference of highorder harmonics.

The analysis drawn in this paper will trigger future experimental demonstrations. Indeed, the FTM effect under phase modulation condition needs to be explored. There is also an intriguing transition from sub-threshold operation, described here, and the laser operation above threshold where repetition rate multiplication has already been observed experimentally (the so-called rational harmonic mode-locking [16,17]). Besides, the AMcase was already investigated in [13], but the present analysis predicts the possibility of observing repetition rate multiplication, which needs further experimental efforts.

\section{Appendix A}

We introduce $f^{(l)}(t)=\bar{\gamma}^{l} \prod_{n=1}^{l} e^{j \omega_{m} t-j 2 \pi n \frac{p}{q}}$. The product is straightforward to calculate and gives $f^{(l)}(t)=\left(\bar{\gamma} e^{j \omega_{m} t}\right)^{l} e^{-j \pi \frac{p}{q}(l+1)}$. Then the relationship between $f^{(l+m q)}(t)$ and $f^{(l)}(t)$ is given by

$$
f^{(l+m q)}(t)=f^{(l)}(t)\left(\bar{\gamma} e^{j \omega_{m} t}\right)^{m q} e^{-j \pi p m(m q+1)}
$$

that we discuss according to the parities of $p$ and $q$. Recall that $p$ and $q$ are coprimes. If $p$ is even (with $q$ odd), $e^{-j \pi p m(m q+1)}=1$ always. If $p$ is odd, $e^{-j \pi p m(m q+1)}=(-1)^{m}$ when $q$ is even, and then $e^{-j \pi p m(m q+1)}=1$ when $q$ is odd. It permits to write $e^{-j \pi p m(m q+1)}=$ $e^{-j \pi m(q+1)}$, which remarkably do not depend on $p$. We then define $\rho \equiv\left(\bar{\gamma} e^{j \omega_{m} t}\right)^{q} e^{-j \pi(q+1)}$ and rewrite

$$
f^{(l+m q)}(t)=f^{(l)}(t) \rho^{m} .
$$

Now suppose $N=K q\left(K \in \mathbb{N}^{*}\right)$. Then Eq. (10) becomes:

$\sum_{l=1}^{q} f^{(l)}(t) \sum_{m=0}^{K-1} \rho^{m}$,

$$
L_{F}(t)=\sum_{l=1}^{N} f^{(l)}(t)=\sum_{m=0}^{K-1} \sum_{l=1+m q}^{q+m q} f^{(l)}(t)=\sum_{m=0}^{K-1} \sum_{l=1}^{q} f^{(l+m q)}(t)=
$$

leading to Eq. 11 :

$$
L_{F}(t)=\frac{1-\rho^{K}}{1-\rho} \sum_{l=1}^{q} f^{(l)}(t)
$$

\section{Appendix B}

Since $\sum_{n=1}^{q} e^{-j 2 \pi n \frac{p}{q}}=0$, by multiplying by the exponential function $e^{j \omega_{m} t}$ one finds $\sum_{n=1}^{q} e^{j \omega_{m} t-j 2 \pi n p / q}=0$. By expanding the sum using Euler's formula, we get $\sum_{n=1}^{q} \cos \left(\omega_{m} t-2 \pi n p / q\right)=0$ and $\sum_{n=1}^{q} \sin \left(\omega_{m} t-2 \pi n p / q\right)=0$. It follows that

$$
\prod_{n=1}^{q} e^{j \delta \sin \left(\omega_{m} t-2 \pi n p / q\right)}=e^{j \delta \Sigma_{n=1}^{q} \sin \left(\omega_{m} t-2 \pi n p / q\right)}=1
$$

Writing $f^{(l)}(t)=\bar{\gamma}^{l} \prod_{n=1}^{l} e^{j \delta \sin \left(\omega_{m} t-2 \pi n p / q\right)}$ and using Eq. (A5), then 


$$
\begin{aligned}
& f^{(l+q)}(t)=\bar{\gamma}^{l+q} \prod_{n=1}^{l+q} e^{j \delta \sin \left(\omega_{m} t-2 \pi n p / q\right)}=f^{(l)}(t) \bar{\gamma}^{q} \prod_{n=l+1}^{l+q} e^{j \delta \sin \left(\omega_{m} t-2 \pi n p / q\right)}= \\
& f^{(l)}(t) \bar{\gamma}^{q}
\end{aligned}
$$

Suppose $N=K q$, ( $K$ integer). Then Eq. (19) becomes:

$$
\begin{aligned}
& L_{F}(t)=\sum_{l=1}^{N} f^{(l)}(t)=\sum_{m=0}^{K-1} \sum_{l=1+m q}^{q+m q} f^{(l)}(t)=\sum_{m=0}^{K-1} \sum_{\mathrm{l}=1}^{q} f^{(l+m q)}(t)= \\
& \sum_{l=1}^{q} f^{(l)}(t) \sum_{m=0}^{K-1} \bar{\gamma}^{m q}=\frac{1-\bar{\gamma}^{N}}{1-\bar{\gamma}^{q}} \sum_{l=1}^{q} f^{(l)}(t),
\end{aligned}
$$

corresponding to Eq. (20).

\section{Funding}

This work was partially funded by Région Bretagne, FEDER (EU), and Rennes Metropole, in the framework of CPER SOPHIE-Photonique. H. Yang was the recipient of a Chinese Scholarship Council (CSC) grant.

\section{References}

1. H. Guillet de Chatellus, O. Jacquin, O. Hugon, W. Glastre, E. Lacot, and J. Marklof, "Generation of ultrahigh and tunable repetition rates in $\mathrm{CW}$ injection-seeded frequency-shifted feedback lasers," Opt. Express 21, $15065-15074$ (2013).

2. H. Guillet de Chatellus, L. R. Cortés, and J. Azaña, "Optical real-time Fourier transformation with kilohertz resolutions," Optica 3, 1-8 (2016).

3. C. Schnébelin and H. Guillet de Chatellus, "Agile photonic fractional Fourier transformation of optical and RF signals," Optica 4, 907-910 (2017).

4. H. Guillet de Chatellus, L. Romero Cortés, Côme Schnébelin, Maurizio Burla, and José Azaña, "Reconfigurable photonic generation of broadband chirped waveforms using a single CW laser and lowfrequency electronics," Nat. Commun. 9, 2438 (2018).

5. H. Yang, M. Brunel, H. Zhang, M. Vallet, C. Zhao, and S. Yang, "RF up-conversion and waveform generation using a frequency-shifting amplifying fiber loop, application to Doppler velocimetry," IEEE Photon. J. 9, 7106609 (2017).

6. V. Duràn, C. Schnébelin, and H. Guillet de Chatellus, "Coherent multi-heterodyne spectroscopy using acoustooptic frequency combs," Opt. Express 26, 13800-13809 (2018).

7. J. Clement, C. Schnébelin, H. Guillet de Chatellus, and C. R. Fernandez-Pousa, "Laser ranging using coherent pulse compression with frequency shifting loops," Opt. Express 27, 12000-12010 (2019).

8. H. Zhang, M. Brunel, M. Romanelli, and M. Vallet, "Green pulsed lidar-radar emitter based on a multipass frequency-shifting external cavity," Appl. Opt. 55, 2467-2473 (2016).

9. H. Yang, C. Zhao, H. Zhang, Z. Zhang and K. Gui, "A novel hybrid TOF/phase-shift method for absolute distance measurement using a falling-edge RF-modulated pulsed laser." Opt. Laser Technology 114, 60-65 (2019).

10. F. V. Kowalski, S. J. Shattil and P. D. Hale, "Optical pulse generation with a frequency shifted feedback laser," Appl. Phys. Lett. 53, 734-736 (1988).

11. H. Sabert and E. Brinkmeyer, "Pulse generation in fiber lasers with frequency shifted feedback," J. Lightwave Technol. 12, 1360-1368 (1994).

12. L. Wang and S. LaRochelle, "Talbot Laser with Tunable GHz Repetition Rate using an Electro-Optic Frequency Shifter," in Conference on Lasers and Electro-Optics, OSA Technical Digest (online) (Optical Society of America, 2017), paper JW2A.66.

13. H. Yang, M. Vallet, H. Zhang, C. Zhao, and M. Brunel, "Pulse doublets generated by a frequency-shifting loop containing an electro-optic amplitude modulator," Opt. Express 27, 18766-18775 (2019).

14. L. P. Yatsenko, B. W. Shore, and K. Bergmann, "Theory of a frequency-shifted feedback laser," Opt. Commun. 236, 183-202 (2004).

15. H. Guillet de Chatellus, E. Lacot, W. Glastre, O. Jacquin, and O. Hugon, "Theory of Talbot lasers," Phys. Rev. A. 88, 033828 (2013).

16. C. Wu and N. K. Dutta, "High-repetition-rate optical pulse generation using a rational harmonic mode-locked fiber laser,” IEEE J. Quantum Electron. 36, 145-150 (2000).

17. Z. Ahmed and N. Onodera, "High repetition rate optical pulse generation by frequency multiplication in actively mode-locked fiber ring lasers," Electron. Lett. 32, 455 (1996). 